\title{
Three Kinds of Normative Behaviour: Minimal Requirements for Feedback Models
}

\begin{abstract}
We present an analysis for modelling social norms. In social psychology three different normative behaviours have been identified: obedience, conformity and compliance. We show that this triad is a useful conceptualisation of normative behaviour and that current models only ever deal with conformity and obedience two, neglecting compliance. We argue that this is a result from modelling having so far focussed too much on agent behaviour rather than agent knowledge and that cognitive models of normative behaviour are needed to capture this third and arguably most interesting normative behaviour.
\end{abstract}

\section{Introduction}

The conundrum of social norms has been the backbone of sociological enquiry from the very start. Social order exists without any central planning or decentralised negotiation, contract or agreement. The conceptualisation of social facts dominated the analysis for the better part of the last century (from Durkheimian origins to the structuralism of the 1960s). Structuralism 
was later rejected as being too deterministic, not leaving enough agency for individuals (see for example critiques by Bourdieu and more recently Coleman (1990)). But with a starting point of individuals alone it is hard to explain the phenomenon of social order. This is commonly known as the structure-agency problem of social norms (e.g. see inter alia Ritzer (2007), Giddens (1993) and Goffman (1959)).

Different types of social norms can easily be distinguished but it is hard to give a fully fledged typology (Verhagen (2007)). There are coordination norms, like driving on the left or right, there are fashion norms, like wearing hats or ties, there are moral norms concerning e.g. abortion rights. In terms of modelling there is an array of models of norms, from game theoretic models conceptualising norms via cooperation and defection (Axelrod (1984)) over models transmitting norms via imitation mechanisms (e.g. Flentge et al. (2001), Gatherer (2002), Epstein (2000)) to models analyising the function of norms (Conte and Castelfranci (1995)). We can categorise norms by content, transmission mechanism, origin, function etc. but it is likely that we will fail like many other categorisations before (Morris (1956)). The reason is that it is not the content of the norm itself that is interesting but how and why people adhere to a norm and for that it does not matter whether it is wearing a hat to a wedding of protesting against an abortion clinic.

Rather than attempting a new typology or categorisation of norms we use a tripartition of normative behaviour from social psychology. There are three essential ways of people adhering to norms: conformity, obedience and compliance. Using these three types of adherence to norms we sort existing models of normative behaviour. We show that compliance has not been captured in any models so far and argue that it is the most interesting adherence type as it alone displays a feature unique to humans: context sensitive 
deliberation about values.

The remainder of the paper is organised as follows. In Section 1 we give a definition of normative behaviour. In Section 2 we explain conformity, obedience and compliance from a social psychology point of view. We particularly focus on compliance, arguing that it is the mechanism of behaviour unique to humans. In Section 3 we sort a set of major models of normative behaviour into the categories established in Section 2. In Section 4 we discuss some minimal cognitive requirements models of normative behaviour have to incorporate if they want to tackle compliance. We conclude in Section 5.

\section{Defining Normative Behaviour}

In order to get a handle on normative behaviour we need to define in some way what we mean by it and how it contrasts with other behaviour. Just like with the typology of social norms, there are many different definitions in the literature on what constitutes normative behaviour. We discussed different origins and definitions of agent-based models of norms research in Xenitidou and Elsenbroich (2010). These different origins put a different emphases on aspects of social norms. For example, game theory stresses the punishment aspect for deviance whilst social physics stresses the imitation aspect of norms (cf. Axelrod (1984), Epstein (2000)). Recent approaches (Andrighetto et al. (2007), Lorscheid and Troitzsch (2009), Lotzmann and Möhring (2009b)) have developed a new angle on normative behaviour which lies somewhere between the punishment and an imitation approach. Here normative behaviour is defined as behaviour resulting from norm invocation, usually implemented in the form of invocation messages which carry the notions of social pressure, but without direct punishment, and the notion of 
assimilating to a social surrounding without blind or unthinking imitation. Although we like this definition we think it is too narrow to capture the complicated range of normative behaviour that exists in human society. We discuss this range in Section 2 and it will be clear that a classification of behaviour as normative via norm invocation messages will not cover the full range.

We want to adopt a definition that is both wide and narrow in some respects, to capture the intuitive range of normative behaviour as well as the range identified in other scientific fields. We bring context and deliberation into the definition, focus on the interconnection between decision and behaviour and juxtapose normative behaviour to behaviour resulting from personal values. For this discussion we assume that a distinction between behaviour resulting from social influence and behaviour independent of social influence can be made.

Definition 1.1 (Normative Decision) A decision is a normative decision if it is brought about by (direct or indirect) social influence.

This means a decision is normative if there is social influence (pressure, invocation, threat of punishment, etc.) on the agent and if the agent makes the decision in favour of the social demand.

Definition 1.2 (Normative Behaviour) A behaviour is normative if and only if it results from a normative decision.

This definition is an "if and only if" meaning that a) all behaviours resulting from normative decisions are normative behaviours and that b) only behaviours resulting from normative decisions are normative behaviours. Statement a) is relatively uncontroversial. If an agent makes a normative decision 
as in Definition 1.1, the resulting behaviour will be normative. b) however excludes from normative behaviour any behaviours resulting not from choice/not involving decision at all. Automatic responses are thus excluded and it can be argued that our definition excludes a vast set of behaviours that should be classified as normative, namely automatic behaviours resulting from internalised norms. On the one hand this means that we see all behaviour resulting from social influence as normative behaviour, which is a wide definition. On the other hand it means that we exclude from our definition all behaviour that is non-deliberative, i.e. automatic behaviour, internalised behaviour etc. which is rather narrow. This is a controversial step as internatlisation is seen as the hallmark of normative behaviour in much of the literature (see Neumann (2009) and references therein). We feel justified to exclude it from our definition nonetheless as we are concerned with deliberative normative behaviour. Once a social norm has become internalised the agent no longer makes a decision about its behaviour. For us, there are three kinds of behaviour: value-led, normative and automatic behaviour; this article is concerned with the first two and excludes the third. Internalisation is the reason for a transition of a behaviour from deliberative normative behaviour to automatic behaviour. Although mechanisms of norm internalisation are an important research topic (see the EmiL Project for great advances on this front ${ }^{1}$ ) it is not our concern in this paper and once a norm has been internalised, the behavioural output becomes uniform (the automatic response) until the next behavioural decision at which point our definitions will apply again.

Let us see with some examples what is covered by our definition. First

\footnotetext{
${ }^{1}$ http://emil.istc.cnr.it/
} 
of all, some deviant behaviour is included in our definition of normative behaviour. A teenager rebelling against a set of social norms might make this decision due to social influence, for example if she rebells simply to rebell against her parents. Here the parents are something to react against. Also, deviant behaviour with respect to a majority norm can easily be normative behaviour with respect to a minority norm, i.e. the teenager rebelling due to her peer-group. Here the peer group is something to behave in accordance with. Depending on the directedness of the decision, the teenager's behaviour may be deemed normative or deviant. This means, whether behaviour is seen as deviant or normative is contextual, dependent on the external evaluation. Secondly, one and the same behaviour can be normative or non-normative. Wearing a hat to a wedding because it is "the done thing" and wearing a hat to a wedding because one just loves hats, or protesting against an abortion clinic because many people are demonstrating against it or because of a deeply held aversion to abortion result in the same behaviours but have different motivations, one normative the other based on individual values and tastes.

Some behaviours that might look like social influence need to be excluded. For example if someone is told about a post at a university by a friend and decides to apply. The decision to apply could not have been made without the friend's input meaning that there was some social aspect to the application in the sense that information was received in the context of a relationship. In itself this scenario does not constitute social influence as all the friend did was to pass on information. However, if the decision to apply for the job was brought about because the friend would be annoyed if the person did not apply and, therefore, treated applying for the post as normative (e.g. because it was a tailor-made internal post, or the information receiving friend 
had been complaining he has been unemployed for a long time), it would be an instance of normative behaviour according to Definitions 1.1 and 1.2. ${ }^{2}$

Another scenario is that of Amy asking Ben to jump off a bridge. If Ben jumps it is a case of behaviour brought about by social influence, but is it normative? According to Definitions 1.1 and 1.2 it is normative behaviour. What if Amy's request was a joke? If Ben jumps it will still be normative behaviour. If Ben does not jump without recognising that Amy's request was a joke Ben might behave according to his own values (such as survival). If Ben recognises that Amy is joking and does not jump we could either interpret his behaviour as normative in the sense that he understands that Amy does not want him to jump or that no decision has to be made in the first place as jumping is not considered an action alternative (i.e. it is not normative behaviour as there is no specific behaviour to be analysed). ${ }^{3}$

For the last two scenarios a classification as normative behaviour might seem counterintuitive. The reason for this is that they do not display something that is usually seen as an important feature of normative behaviour: being a behavioural regularity in a population. Although an important macroaspect of normative behaviour it cannot work as a definition of normative behaviour. The problem is essentially a limit problem. To be a behavioural regularity in a population we need to define what it means to be a regularity and what is a relevant population. How often does a behaviour have to be repeated in order to be a regularity? And how many people make a population? In the limit the first question might be answered by saying that a behaviour has to be displayed at least twice, i.e. one repetition for it to make a regularity (any other number would be arbitrary). However, we

\footnotetext{
${ }^{2}$ Thanks to an anonymous referee for this scenario.

${ }^{3}$ Thanks for this scenario to an anonymous referee.
} 
would not describe a behaviour repeated once as a behavioural regularity. Similarly with the population size. If we take a whole population (a 'nation', an 'ethnicity', a 'culture') we will not find a single behaviour displayed by everyone. We thus have to divide the population up. Further we cannot take population majorities as indicative of normative behaviour as we need to do justice to minority norms. In the limit a population could be defined as two people. We end up with a behavioural regularity in a population as 'the same (similar?) behaviour executed twice or by two people' which is just as counterintuitive as our definition above appears to be.

These two limit problems are less of a problem when wanting to identify normative behaviour, we look at some population and look for some regularities, i.e. we carve the population up into sub-populations that display similar behaviour types (although even this categorisation needs plenty to background knowledge to rightly group behaviours). Thus, being a behavioural regularity in a population is a useful description of normative behaviour but not a definition.

Finally, not all behavioural regularities in a population are normative. In the UK, the Pharmacy "Boots" gives away free nappy changing bags to members of its Parenting Club. As a result, every second pram on the road is adorned by the same grey and black bag. This is a behavioural regularity but it's origin can hardly be called normative.

We think that a pure macro-definition of normative behaviour as 'a behavioural regularity in a population' is thus untenable. ${ }^{4}$ Instead of the behavioural regularity aspect we focus on how agents adhere to norms, i.e.

\footnotetext{
${ }^{4}$ Incidentally, the definition used in 'EmiL' where normative behaviour is defined as behaviour coming about by norm invocation does not pay heed to the behavioural regularity definition.
} 
whether they conform, obey or comply which means the focus is on the individual agent with the population regularity as an emergent phenomenon, i.e. if enough people ask their friends to jump off bridges and they do so, the result is a social norm of bridge jumping.

In the following section we discuss the distinction between three kinds of normative behaviour from social psychology to give a theoretical embedding of our definitions.

\section{Social Psychology}

Social psychology is a theoretically rich and varied social science discipline and any given categorisation of norms will be severely contested by respective opponents. Nonetheless, the categorisation of normative behaviour into conformity, obedience and compliance has a relatively wide reach within the theoretical landscape (see inter alia Xenitidou and Elsenbroich (2010), Franzoi (2000), Myers (2002), Hewstone et al. (2008)). The categorisation is based on the conclusions of studies in experimental social psychology which dealt with social norms focusing on the ways in which individuals influence one another in group situations (Sherif (1936); Asch (1952); Milgram (1974)). Henceforth, conformity, obedience and compliance have been described as the behavioural consequences of social influence.

The first way of norm adherence is conformity. An agent conforms if it simply follows the behaviour of other agents. An agent can conform to a majority or a subgroup of the population. We will drive on the left hand side of the road when in Great Britain but on the right on the European continent. We might wear bumbags and socks when on holiday in Italy just like other tourists or wear sunglasses and black like the Italians do. Both 
mean we conform to a subset of the population.

Conformity just means an agent has some behaviour that it will change and adapt to the behaviour prevalent in a group (often but not necessarily the majority of the population) (see Asch (1952)). The adoption of the norm is complete once the agent becomes aware of the group behaviour meaning that nothing specific to the individual agent needs to be carried over to the post-norm adoption state. The notion of conformity can be linked to structuralism as it shows the way social structure influences an individual's behaviour. The problem of structuralism is that it leaves no room for the individual, making agents nothing but social puppets, normalised through imitation and, thus, thoughtless (on automatic or thoughtless conformity see Aarts and Dijksterhuis (2003); Dijksterhuis and Bargh (2001)).

Seeing conformity as "unthinking" adherence to a socially demanded behaviour seems to make it not covered by our definition. Although once the behaviour is adopted the agent conforms unthinkingly, there is usually a point at which an agent chooses to conform or not. If there is such a choice-point, and the agent changes its behaviour to conform with normative demands, the behaviour will result from a normative choice and thus be normative behaviour. Our definition does not apply to cases where there is no choice point.

The crucial distinction that we thus aim to make is between making a decision according to individual values and a decision according to (direct or indirect) social influence. We are aware that this sharp demarcation is potentially problematic. We want to make the demarcation nonetheless as it will help us to clarify what is missing from agent-based modelling of normative behaviour. We loose out on some aspects of normative behaviour this way, like internalisation etc. but we think that in terms of modelling 
the interesting aspect of normative behaviour is how agents change their behaviour rather than how they keep behaving in one way thus making the choice-points crucial rather than the internalisation.

The second way of norm adherence is obedience. An agent obeys a norm if adherence to a norm result from an authority enforcing the norm, e.g. by punishment. The punishment can be applied by a small group or even a single person with authority, i.e. the police or a father. Punishment can also be applied by the agent's community, in the form of ostracism for example. We will not jump a red light in the car because we are afraid of being caught and fined by the police.

Like conformity, obedience only demands the agent to make a binary choice. If the external pressure becomes too big the agent will change its behaviour and obey the norm. The agent needs to understand the cost of defying a norm, i.e. the possibility and severity of punishment. Nothing changes in the agent between the pre-norm adoption and post-norm adoption state (see Milgram (1974) and Adorno et al. (1950)). Norm adherence is fully determined externally. Analyses of norms using the mechanism of obedience are theories based on a Hobbesian conception of society constraining the individual, such as Rational Choice theory and game theory (e.g. Coleman (1990), Bicchieri (2006)). These theories, relying on individualism have been accused of not taking the social influence on agents seriously.

One might think that obedience is simply a case of self interest, covered by any theory of cost benefit analysis. ${ }^{5}$ Although the decision to act in a certain way is reached by weighing up the cost and benefit of the punishment versus the action, the case of obedience is a special subset of general cost-

\footnotetext{
${ }^{5}$ Thanks to an anonymous reviewer for pointing at this potential problem.
} 
benefit analysis as the demand to act in a certain way is socially induced and contains the notion of punishment which carries with it a notion of authority and hierarchy not present in other cost-benefit analyses. So although we can say that a case of obedience is a cost benefit analysis, not every cost benefit analysis is obedience.

The third way of norm adherence is compliance. An agent complies with a norm if it follows it whilst being able to consider other behaviours. To comply contains the element of decision, to comply to something now but not later or to comply to something superficially but not really. Often compliance is associated with publicly acting in a certain way whilst retaining ones personal values. We might comply with wearing a suit to work although we would never wear formal clothes otherwise.

Compliance is a more complex form of norm adherence than conformity and obedience. Conformity means the agent changes its behaviour continuously, i.e. once conformity has occurred it does not go back to previous behaviour. The new behaviour becomes the only behaviour the agent's repartoire. Obedience is enforced behaviour in which the agent does not actually change its desires but suppresses them due to threatened punishment. Once the threat is removed the agent can go back to its "old ways".

Whilst in conformity the change in the agent is complete and in obedience there is essentially no change at all, compliance contains the possibility of change in some instances given that there is some concession of the individual to social influence and internal pressure whilst keeping their individual 'values/desires' alive. Compliance is also the form of norm adherence that is most genuinely human. Whilst animals display conformity behaviour (flocking, ant societies, etc.) and obedience (dog training etc.) they do not 
display context dependent behaviour resulting from compliance. Compliance demands representations of the world 'given compliance' and the world 'without compliance' and behaviour choices and decisions sensitive to the context. It also demands intentionality; the agent decides whether to comply to a norm or not intentionally. The interplay of these representations, the intentionality and choices is where the interesting feedback between the individual and society lies.

How do these three kinds of normative behaviour relate to our definitions of normative decision and normative behaviour? Each one is about a behaviour change but each one relates to a slightly different kind of social influence and a different kind of information informs the agent's decision. To conform means to change ones behaviour to conform to a majority social norm. The decision is informed by a calculation of the number of the agents displaying the behaviour and according adaptation. To obey means to change ones behaviour in the face of possible punishment. This decision is informed by the calculation of utilities for the agent. Is the cost of punishment higher than the benefit of sticking to my values? If this is answered yes the agent will change its behaviour. To comply means to prioritise public values over ones own values, i.e. make a normative decision, but the information is neither simple popularity of the normative position nor the threat of punishment. The information is about the behaviour itself and social reasons for foregoing individual values. An agent might comply because it assesses the particular behaviour as good or appropriate in a particular context; for example, because it has contractually bound itself to some behaviour or it might comply because it wants to please someone else, a parent/friend/spouse/child/employer. These considerations are rather different 
from those of conformity and obedience. Only in compliance we prioritise public over individual values whereas in conformity we make a simple calculation of popularity and for obedience we calculate the benefit and cost of an action for us, without any consideration of others. And only compliance leaves the possibility of complying with a norm in one context and acting according to ones own values in another.

However, all three are behaviour changes due to social influence meaning that our definition captures all three kinds whereas other definitions capture only one or maybe two aspects, see Xenitidou and Elsenbroich (2010).

\section{Models of Normative Behaviour}

In this section we use the above categorisation and relate it to existing agentbased models of normative behaviour. We argue that historically agent-based modelling has solely focussed on conformity and obedience. As a result, agent-based models have not yet achieved their potential to model normative behaviour.

Agent-based modelling has its origin in several disciplines of both the natural and social sciences, e.g. game-theory, artificial intelligence, (social) physics. ${ }^{6}$ Many agent-based models of normative behaviour take their starting point in game theory building on the original work by Axelrod (1984, 1986). Here normative behaviour is modelled as a binary concept: the agent has the two behaviour options to cooperate or defect. Cooperation, in this account is normative behaviour. Agents are defined as utility maximising or satisficing (Simon (1982)) using an agent specific utility function. The first assumption of game theory is that cooperation is costly for the agent, more

\footnotetext{
${ }^{6}$ For an excellent classification of models see Neumann (2008).
} 
costly than defection. The model then tries to skew the utility function of the agents towards cooperation, using severity of punishment and the likelihood of being caught as the variables. The goal is to find the mechanisms that let cooperation emerge from the presupposed selfish, defective behaviour of agents. In Axelrod (1986), for example, a surprising outcome was that cooperation does not emerge with simple punishment but that agents that do not punish an observed defection of a norm need to also be punished. Only with this meta-norm game the utility function be skewed enough to ensure cooperation. Models resulting from game theory can be collectively classified as models of obedience. All of them use the enforcement of sanctions (punishment) as the mechanism that makes people adhere to norms (cooperate). There are variations on the theme of game theory. For example, other agents might not directly punish defection but punishment might be exerted by loss of reputation (Hales (2002)), loss of trust (Macy and Skvoretz (1998)) or ostracism (de Pinninck et al. (2008)).

Another notable strand of agent-based models of normative behaviour relies on imitation mechanisms. Norms are again characterised as binary choices although there are less assumptions in these models concerning the utility of either norm to an agent. Most of the time the two norms are symmetric in value. Epstein (2000) for example gives a model of normative behaviour in which people switch their behaviour depending on the agents surrounding them. People want to 'fit in' in this model. In addition to this he formalises the behaviour that people, when they do 'fit in', get lazy and stop thinking about their behaviour. Thus conformity obviates the need for thinking and agents check less agents around them on fitting in. Other models of this kind are Flentge et al. (2001) and Gatherer (2002). These 
models use memes ${ }^{7}$ and imitation mechanisms for the spread of normative behaviour among agents. Models using imitation are conformity models of normative behaviour. An agent gives up its behaviour completely if a large enough number of the population displays a different behaviour; behaviour change is due to direct or indirect majority or group pressure.

We think that most agent-based models of normative behaviour fit into these two categories. Exceptions from the classic models of normative behaviour are Conte and Castelfranci (1995) and Castelfranchi et al. (1998). These models are of a different kind as they assess the function of norms for the wellbeing of the society rather than adherence to norms.

We mentioned above that agent-based models to date have only captured obedience and conformity. We think the reason for this is that both concepts can be modelled using simple calculation, as has been described above in the Epstein model for conformity and the Axelrod model for obedience. To model compliance we need deliberation about personal and public values. In the next section we present what we see as the first steps towards models of norm compliance.

\section{Compliance Models}

Let us come back to compliance defined as public conformity whilst keeping personal values. This implies two levels of conceptualisation, a private and a public one. The agent needs to a) have a belief base of its personal attitudes, values and beliefs about the world. It b) needs to have a representation of the external world including the understanding of normative rules and

\footnotetext{
${ }^{7}$ The concept of meme goes back to Dawkins (1976) and is the unit of selection in cultural selection just like the gene in natural selection.
} 
judgements. And finally c) it needs a way of combining those two, i.e. of judging, choosing, integrating and juxtaposing the internal and external.

So far we have established that compliance is not covered as a normative behaviour in agent-based modelling and that it is arguably the most interesting as it is uniquely human. The explanation for this omission is simple: conformity and obedience fit into the paradigm of agent-based modelling of focussing on behaviour (rather than cognition). For conformity it is sufficient to have a rule that a behaviour is changed if a different behaviour is displayed in a certain number of other agents (threshold) in a certain environment (neighbours, whole population etc.). The agent needs a factual representation of the outside world to know when to change behaviour but it needs no normative knowledge whatsoever. For obedience the behaviour of an agent changes when a behaviour is "punished". This punishment needs a value/utility assignment to the behaviour and a value/utility assignment to the punishment. The utility of the behaviour is assessed by the agent by subtracting the punishment assignment from the behaviour assignment. The behaviour is changed if the utility becomes too low. In order to achieve compliance we need agents that can make decisions sensitive to contexts for which an agent needs to be able to reason about its own values and external normative demands.

The standard agent architecture for normative behaviour is the BOID agent (Dignum et al. (2002)), an architecture implementing the interaction between beliefs, obligations, intentions and desires. The beliefs are the connector to the outside world, they are facts. Obligations are a set of defeasible rules the agent should follow. Intentions are plans resulting from the interplay of the other three ingredients and desires are what the agent wants to 
achieve, its goal. There is a clear distinction between factual and normative as they are located in distinct compartments of the architecture. This however means that the interface with the world is here only defined as factual with the normative obligations being constant or static. As a consequence there is no feedback between the outside world and the normative part of the agent architecture. There exists a host of literature starting from Alchourrón et al. (1985) how internal external interaction is handled for factual statements. The literature of belief revision is all about how inconsistency is dealt with and how new knowledge changes the internal belief base. The interesting point about compliance is that there is no immediate change to the belief base, i.e. the internal belief base is retained whilst external behaviour is displayed. Change to the internal belief base (which here crucially includes normative beliefs) can occur gradually through the constant interaction with the external (normative) world.

There are two modelling approaches we want to discuss as promising starting points of compliance models. One is recent work on the feedback between society and individuals by norms captured in the EmiL-project (Andrighetto et al. (ming) (forthcoming)) the other is work on the learning of group norms by Verhagen (Verhagen (2001)).

\subsection{Emergence in the Loop - EmiL}

One of the first agent architectures concerned with norm adaptation and innovation is the EmiL-A architecture first developed in Andrighetto and Campenni (2007). The starting point here is a division of agent knowledge into factual (event) and normative (rule) knowledge. The agent now has a normative interface with the world rather than just the factual one of the BOID agent. A simulation of the feedback mechanism between norms and 
agents, building on a simplification of the above architecture is described in Lotzmann and Möhring (2009a,b) and implemented in Lotzmann et al. (2008) for the case of pedestrian-driver interaction in traffic situations. The agents in a simulation have two kinds of memories, an event board, which stores facts and events and a normative frame there to infer and store rules from the event board. In addition agents are informed of norms that have emerged communally by the normative board where this common information is stored. The focus here is on how agents understand, learn and change norms by adaptation and invocation. Lorscheid and Troitzsch (2009) explore learning concepts for this new kind of information and test them in a norm invocation model. The authors identify reinforcement learning as one underlying mechanism but insufficient to model norms. They add normative learning which is learning from normative invocation messages sent between agents. The mechanism of norm invocation messages incorporates a penitance level, a threshold for individual agents at which they change their behaviour and a social hierarchy, by having authority levels of agents matter for norm adherence.

In this model agents have a personal value and are confronted with public values in the form of norm invocations. The penitence level means that agents do not just conform blindly to public demands but deliberate upon them.

According to the EmiL architecture (EmiL-A), this deliberation results in behavioural outputs which could take the form of two different kinds of normative actions, compliance/violation and norm-defence. We think that compliance as used here differs from our understanding of compliance and falls within our definition of conformity. This is because while there is a decision-making process which consists of checking against potential obstacles (material impossibility and goal conflict) of conforming to a norm or not, 
this takes place providing a norm has been recognised as such and adopted. At that stage then, the action opted for is either a result of instrumental reasoning or cooperative/normative disposition rather than situational (and the norm enters the normative board, an archive in the long-term memory where active norms are stored and arranged according to their salience, i.e. degree of activation). EmiL-A, therefore, seems to allow for a form of contextbased reasoning for whether to conform to a norm or not, (see Andrighetto et al. (ming)), (while EmiL-S (see Lotzmann (2010)) allows for a form of deliberation comparing the current situation to an earlier one in which the agent received a norm invocation. The similarity of the current and the former situations determines the cogency of alternative norms).

Despite the possibility of context sensitive normative behaviour, our understanding of compliance significantly differs in that it makes normative action possible irrespective of whether it is backed up by the normative belief - normative goal norm adoption process. Rather, the process underpinning compliance is one of comparison of personal to social beliefs/values in a given situation, which could result in acting in accordance with but not (necessarily) adopting a norm. Therefore, our understanding of compliance enables breaking away from the interconnection between belief-decision-behaviour (and, thus, from the longstanding problem of linking attitude to behaviour) and focuses on the interconnection between decision and behaviour instead, thus allowing for the explanation of a wider range of normative behaviours that exist in human society.

We believe that the different understandings of compliance result from the line of reasoning underpinning EmiL-A being influenced by a functionalist approach to norms as functional constituents of social order. 


\subsection{Group Norms and Learning}

Verhagen (2001) develops a model for the learning of group norms. Agents are set in a two-dimensional world with two resources, Resource A and Resource B. Patches of the world can contain nothing, one of the resources or both resources. Agents can decide to consume one of the resources, move to another patch or do nothing. Agents base their decisions on two decision trees, a personal one and a group one. The personal decision tree is based on the utility the agent gets from the action. The group decision tree represents the agents interpretation of the group norms. For an agent's decision the trees are combined to one single tree and the action having the combined highest utility is chosen by the agent. Subsequently the agent receives feedback from the group on its decision resulting in an update of the group decision tree.

Although we have the two layers of a personal value sphere and the group norms (as perceived by the agent), this is not a model of compliance. ${ }^{8}$ The action decision is not made weighing up the internal values and external demands with behaviour being adapted case by case. The model is a model of the learning of norms. The agent receiving feedback on its decisions using the combined decision tree can update what it understands the group norms to be. It does, however, evaluate the group norms independently of its own norms, making a decision whether to comply with the group norms or follow its personal benefit. However, this model, by virtue of having the public and private sphere of norms and values (or in this case utilities), the model can serve as a starting point for a model of compliance. What needs to be adapted is the evaluation of the two decision trees to allow agents to

\footnotetext{
${ }^{8}$ Thank you to an anonymous reviewer for suggesting this model.
} 
deliberate between them (rather than calculate an overall utility).

\subsection{Compliance Model Sketch}

What do we need for a model of compliance? We need an agent architecture that is not only able to reason with norms as is the case in both the EmiL-A architecture and the Verhagen model of norm learning but an agent architecture that allows the agent to reason about norms. In modelling compliance the question almost changes from 'how do agents behave normatively' to 'how do agents violate norms'. The agent has to be able to compare personal values with normative demands and make a decision in a specific situation. The Verhagen model has an important underlying formalisation in that it models

private/individual values and public/group values separately but currently agents do not decide whether to follow one or the other according to a specific situation but according to how independent an agent is in its thinking from the group. The EmiL-A architecture has as an important feature the normative board which allows the learning of norms by interaction with the outside world and can be the underlying 'database' of normative demands an agent perceives. A model of compliance needs in addition something like a value board as a database of personal values (morals) and the agent needs to be able to reason about both boards and their applicability to specific situations.

\section{Conclusion \& Future Work}

We gave a definition of normative behaviour, using the concept of normative decision followed by the discussion of a tripartite of kinds of normative 
behaviour extracted from social psychology. Using the triple distinction of conformity, obedience and compliance, we contend that historic agent-based models of normative behaviour only formalised conformity and obedience. We argued that the most interesting adherence to norms, however, is compliance because it is the uniquely human normative behaviour having the element of deliberation, in contrast to obedience and conformity also being displayed in animals. We gave some examples of normative modelling which, in our opinion go beyond the conformity/obedience frameworks but argue that they do not encapsulate compliance yet. We think that a focus on the theoretical construct of compliance and an embedding of agent-based models into social psychology theories of compliance has pointed at what is missing in the models so far but also points at future developments, such as the incorporation of intentionality and context sensitivity.

Our analysis falls short on two points. First we have the shortcoming that automatic responses to internalised norms do not fall under our definition of normative behaviour. The reason is that we would like to keep normative behaviour as deliberate behaviour and automatic responses are not deliberate. We are thus making a distinction between automatic behaviour, normative behaviour and behaviour following individual values.

Connected to that problem is the fact that often individual values are socially determined through norm internalisation, which is often seen as one of the most important features of normative behaviour, cf. Neumann (2009). Although a weakness, we think that a focus on a definition of normative behaviour on deliberative decision making is useful for agent based modelling as attention will be drawn away from simple calculations, resulting in models of conformity and obedience.

The reason why automatic behaviour is not covered in our approach is 
that we defined normativity using the motivation of the individual agent for the behaviour as the demarcation criterion. This can only be done if the agent makes a decision between individual values and normative behaviour, i.e. deliberates. One way to extend the definition now is to define all automatic behaviour as normative behaviour. We think that this definition will lead into problems as it would let too much behaviour be covered by the label normative. In addition it would require a separate amendment to model the dynamics of social influence. We propose that in order to include automatic behaviour into the analysis of normative behaviour and make our account dynamic,the vantage point should be located outside the agent and into the social and situational. This draws on intersubjectivity and relationality (cf. Garfinkel (1984); Gergen (2009) according to which meaning is obtained via the interactions and interrelations of agents and is contextsensitive and situation-specific. This would mean that, a) for non-deliberative behaviour, whether it is normative or not is decided by the 'audience' of the behaviour and b) that the perception/uptake of an action as normative or non-normative (besides and beyond the origin of the agent's own decision) will feed back into the agent's consciousness for the next decision. We will explore the usefullness of this definition extension in future work.

A second shortfall is that our account has no need for normative behaviour to be rules followed by a population but that behaviour can be normative on a single instance (the example of friends asking an agent to jump off a bridge). We would like to make the distinction between individuals behaving normatively and the existence of social norms, the first being a microproperty, the second a macro property. Our definition is only concerned with the micro-property of normative behaviour. Social norms are the result of enough agents displaying the same normative behaviour. Future work is to 
define an agent architecture able of norm compliance as well as conformity and obedience to investigate the macro phenomenon of social norms.

\section{References}

Aarts, H. and Dijksterhuis, A. (2003). The silence of the library: Environmental control over social behavior. Journal of Personality and Social Psychology, 84.

Adorno, T., Frenkel-Brunswik, E., Levinson, D., and Sanford, R. N. (1950). The authoritarian personality. New York: Harper.

Alchourrón, C. E., Gärdenfors, P., and Makinson, D. (1985). On the logic of theory change: Partial meet contraction and reveision functions. Journal of Symbolic Logic.

Andrighetto, G. and Campenni, M. (2007). On the immergence of norms: a normative agent architecture. In AAAI Symposium, Social and Organizational Aspects of Intelligence.

Andrighetto, G., Campennì, M., and Conte, R. (forthcoming). Minding Norms I: The Foundations. Oxford Series on Cognitive Models and Architectures.

Andrighetto, G., Conte, R., and Turrini, P. (2007). Emergence in the loop: Simulating the two way dynamics of norm innovation. In 07122 , D. S. P., editor, Normative Multi-agent Systems.

Asch, S. E. (1952). Social Psychology. New Jersey: Prentice-Hall.

Axelrod, R. (1984). The evolution of cooperation. Basic Books: New York, NY.

Axelrod, R. (1986). An evolutionary approach to norms. American Political Science Review, 80(1095-1111). 
Bicchieri, C. (2006). The Grammar of Society: The Nature and Dynamics of Social Norms. Cambridge University Press.

Castelfranchi, C., Conte, R., and Paolucci, M. (1998). Normative reputation and the cost of compliance. Journal of Artificial Societies and Social Simulation, $1(3)$.

Coleman, J. S. (1990). Foundations of Social Theory. Harvard University Press.

Conte, R. and Castelfranci, C. (1995). Understanding the functions of norms in social groups through simulation. In Gilbert, N. and Conte, R., editors, Artificial Societies. The computer simulation of social life. London: UCL Press.

Dawkins, R. (1976). The Selfish Gene. Oxford University Press.

de Pinninck, A., Sierra, C., and Schorlemmer, M. (2008). Distributed norm enforcement via ostracism. Lecture Notes in Computer Science, (4870):301-315.

Dignum, F., Kinney, D., and Sonenberg, L. (2002). From desires, obligations and norms to goals. Cognitive Science Quarterly, 2(3/4).

Dijksterhuis, A. and Bargh, J. A. (2001). The perception-behavior expressway: Automatic effects of social perception on social behavior. Advances in Experimental Social Psychology, 33:1-40.

Epstein, J. (2000). Learning to be thoughtless: Social norms and individual computing. Technical report, Center on Social and Economic Dynamics Working Paper, No. 6.

Flentge, F., Polani, D., and Uthmann, T. (2001). Modelling the emergence of possession norms using memes. Journal of Artificial Societies and Social Simulation, 4(4). 
Franzoi, S. L. (2000). Social Psychology. New York: Brown \& Benchmark, 2nd edition.

Garfinkel, H. (1984). Studies in Ethnomethodology. Polity Press Cambridge UK.

Gatherer, D. (2002). Identifying cases of social contagion using memetic isolation: comparison of the dynamics of a multisociety simulation with an ethnographic data set. Journal of Artificial Societies and Social Simulation, 5(4):5.

Gergen, K. (2009). Relational Being: Beyond Self and Community. Oxford University Press.

Giddens, A. (1993). New Rules of Sociological Method. Cambridge: Polity Press.

Goffman, E. (1959). The Presentation of Self in Everyday Life. New York: Doubleday.

Hales, D. (2002). Group reputation supports beneficent norms. Journal of Artificial Societies and Social Simulation, 5(4).

Hewstone, M., Stroebe, W., and Jonas, K., editors (2008). Introduction to social psychology : a European perspective. Oxford: Blackwell, 4th edition.

Lorscheid, I. and Troitzsch, K. G. (2009). How do agents learn to behave normatively? machine learning concepts for norm learning in the emil project. In Edmonds, B. and Gilbert, N., editors, Proceedings of the Sixth Conference of ESSA.

Lotzmann, U. (2010). Enhancing agents with normative capabilities. In Bargiela, A., Ali, S. A., Crowley, D., and Kerckhoffs, E. J., editors, Proceedings of the 24th European Conference on Modelling and Simulation.

Lotzmann, U. and Möhring, M. (2009a). Simulating norm formation - an operational approach. In Decker, Sichman, S. and Castelfranchi, editors, Proc. of 
8th Int. Conf. on Autonomous Agents and Multiagent Systems (AAMAS 2009), pages $1323-1324$.

Lotzmann, U. and Möhring, M. (2009b). Simulating normative behaviour and norm formation processes.

Lotzmann, U., Möhring, M., and Troitzsch, K. G. (2008). Trass - a multi-purpose agent-based simulation framwork for complex traffic simulation applications. In Bazzan, A. L. C. and Kl'ugel, F., editors, Multi-Agent Systems for Traffic and Transportation. IGI Global.

Macy, M. W. and Skvoretz, J. (1998). The evolution of trust and cooperation between strangers: A computational model. American Sociological Association, 63:638-660.

Milgram, S. (1974). The perils of obedience. Harper's Magazine.

Morris, R. T. (1956). A typoloty of norms. American Sociological Review, 21:610613.

Myers, D. G. (2002). Social Psychology. New York: McGraw-Hill, 7th edition.

Neumann, M. (2008). Homo socionicus: a case study of simulation models of norms. Journal of Artificial Societies and Social Simulation, 11(4).

Neumann, M. (2009). Dissecting the boid perspective on norms. In Edmonds, B. and Gilbert, N., editors, Proceedings of the Sixth Conference of ESSA.

Ritzer, G. (2007). Sociological Theory. McGraw Hill, 7th edition.

Sherif, M. (1936). The Psychology of Social Norms. New York: Harper and Raw Publishers.

Simon, H. A. (1982). Models of Bounded Rationality. Cambridge: MIT Press. 
Verhagen, H. (2001). Simulation of the learning of norms. Social Science Computer Review, 19(3):296-306.

Verhagen, H. (2007). Normtypographies. In Boella, G., van der Torre, L., and Verhagen, H., editors, Normative Multi-agent Systems, number 07122 in Dagstuhl Seminar Proceedings, Dagstuhl, Germany.

Xenitidou, M. and Elsenbroich, C. (2010). Construct validity of agent-based simulation of normative behaviour. The International Journal of Interdisciplinary Social Sciences. 